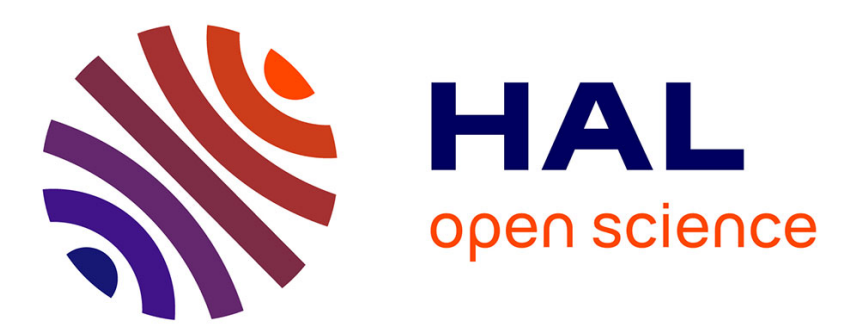

\title{
Hacker la démocratie taïwanaise : Audrey Tang et la réinvention de la politique
}

Emilie Frenkiel

\section{To cite this version:}

Emilie Frenkiel. Hacker la démocratie taïwanaise : Audrey Tang et la réinvention de la politique. Participations - Revue de sciences sociales sur la démocratie et la citoyenneté, 2017, Participer dans le monde chinois : une jeunesse connectée, 17, pp.121-153. 10.3917/parti.017.0121 . hal-01876057

\section{HAL Id: hal-01876057 https://hal.science/hal-01876057}

Submitted on 18 Sep 2018

HAL is a multi-disciplinary open access archive for the deposit and dissemination of scientific research documents, whether they are published or not. The documents may come from teaching and research institutions in France or abroad, or from public or private research centers.
L'archive ouverte pluridisciplinaire HAL, est destinée au dépôt et à la diffusion de documents scientifiques de niveau recherche, publiés ou non, émanant des établissements d'enseignement et de recherche français ou étrangers, des laboratoires publics ou privés. 


\title{
Hacker la démocratie taïwanaise : Audrey Tang et la réinvention de la politique
}

\author{
> Émilie Frenkiel
}

\section{> Résumé}

Cet article est consacré à Audrey Tang, appartenant au collectif de jeunes hackers citoyens g0v et devenue ministre du Numérique à Taïwan en octobre 2016. Il étudie comment les dispositifs numériques de participation sont fabriqués dans le contexte taïwanais, au croisement des influences occidentales et de l'influence de son voisin chinois, et engagé dans un processus de profond renouvellement politique depuis l'occupation du Parlement par des étudiants lors du mouvement des Tournesols de 2014. L'article enquête sur les raisons d'une rencontre originale entre savoir-faire technique, réflexion théorique et volonté politique. 
"La vitesse à laquelle le public forme des liens de confiance mutuelle sur Internet est de loin supérieure à celle du processus décisionnel traditionnel. Si les gouvernements ne font pas de place à ces voix qui s'élèvent, il deviendra impossible de convaincre le public d'accepter ces décisions » (Tang, 2016a)'

"Avant, la démocratie était un affrontement entre deux valeurs opposées. Maintenant, la démocratie est une conversation entre beaucoup de valeurs diverses » (Tsai Ing-Wen, 2016).

"Puisque les hackers citoyens détruisent la vieille politique, il ne reste plus qu'à trouver comment en reconstruire une nouvelle » (Y.-C. Chen, 2016).

Dans son discours inaugural du 20 mai 2016, Tsai Ing-wen, la première femme élue à la présidence de Taïwan, s'est adressée avant tout à la jeunesse taïwanaise. En janvier 2016, le Parti démocrate et progressiste len anglais, Democratic Progressive Party, ci-après DPP), parti indépendantiste taïwanais, a en effet largement remporté les élections présidentielles et parlementaires, et ce certainement en grande partie parce qu'à travers la candidate, il est parvenu à se remettre de la fin de mandat désastreuse en 2008 de l'unique président qu'avait connu le DPP, Chen Shui-bian, et à séduire les jeunes générations (Hsiao, 2016 ; Chan, 2016). Si, chose rare, les trois quarts des 20-29 ans ont en effet participé au vote, ce n'est pas seulement parce qu'elle a opté pour un style dépoussiérant, notamment en mettant en scène ses chats ou avec des clips de campagne conçus par des stars de l'animation, c'est certainement car Tsai est parvenue à incarner une nouvelle direction politique et une profonde réforme des institutions taïwanaises. Elle a promis pendant sa campagne - et renouvelle cette promesse en clôture de son discours inaugural - de répondre aux frustrations des jeunes Taïwanais et d'insuffler une politique nouvelle, notamment vis-à-vis de la Chine, et globalement plus démocratique et fidèle aux désirs de la population. Elle marque ainsi le contraste avec la gouvernance élitiste et opaque des dirigeants du Kuomintang si radicalement dénoncée par le mouvement des Tournesols ${ }^{2}$ en 2014 et à l'origine du militantisme de la hackeuse citoyenne qui fait l'objet de cette étude, Audrey Tang, nommée ministre du Numérique pendant la rédaction de cet article.

En 2012, un petit groupe de hackers commence à faire parler de lui dans la polémique consécutive à la publication d'une vidéo postée sur YouTube par le gouvernement. Censé rendre plus accessible aux citoyens ordinaires un plan de relance économique, le clip vidéo de quarante secondes présente quatre citoyens

[1] Sauf indication contraire, toutes les citations traduites du chinois ou de l'anglais sont de l'auteur. [2] Il s'agit d'un mouvement étudiant qui aboutit, au printemps 2014, à l'occupation du Parlement taïwanais, voir ci-après. 
visiblement dépassés par la complexité de la réforme, dont le nom plane au-dessus de leurs têtes hagardes. Une voix off explique alors que ce serait une perte de temps de chercher à expliquer avec des mots simples le plan de relance du fait de sa complexité : "Il est préférable de se retrousser les manches et de se mettre au travail » (Wang, Shih, 2012). Lorsqu'ils commanditent puis approuvent cette publicité, les dirigeants du Kuomintang ne pressentent pas les frémissements de la Toile que provoquera cette énième ode à la sagesse des dirigeants, au respect de l'autorité, au dur labeur et à l'efficacité. La réaction des internautes annonce, selon Audrey Tang, qu'immergés dans une «société Internet fondée sur la rapidité, la confiance, l'égalité et le partage », la routine gouvernementale du KMT leur est devenue insupportable. Souhaitant défendre l'idée que le gouvernement est à tout le monde, quatre hackers lancent un système d'audit citoyen du budget du gouvernement central. L'idée, issue d'un hackathon, est de rendre les données du Bureau des comptes et statistiques accessibles sur un site Internet interactif et intuitif ${ }^{3}$, et de permettre ainsi au public de noter et de commenter chaque section du budget. L'une des devises de ces programmeurs est «fork the government » : leur objectif est de construire pour chaque extension des sites Internet du gouvernement (terminant par .gov.tw) une bifurcation (.g0v.tw) en open data ${ }^{4}$, plus transparente, accessible, intuitive et surtout participative. Deux mois plus tard, Audrey Tang rejoint le mouvement, qui se destine à créer des outils sur Internet pour la société civile. G0v (lire gov-zero) est nés.

Comme le rappelle avec insistance Audrey Tang lors de notre premier entretien, en anglais, to hack signifie «travailler le bois »; on appelle hackers ceux qui fabriquent eux-mêmes leurs outils, retravaillent ceux qui existent pour les améliorer ou les adapter à leurs nouveaux besoins. Audrey Tang ne prétend pas parler pour l'ensemble de la communauté g0v.tw, à laquelle plus de 100000 personnes ont déjà contribué. L'étude de son parcours, sa vision de la démocratie, ses valeurs et ses actions militantes au sein de g0v offre néanmoins une clé de compréhension stimulante des hacktivistes qui, comme elle, mobilisent les outils, les compétences et aussi les valeurs des hackers au bénéfice de la société et de la démocratie taïwanaises et mondiales (Ermonshina, 2016 ; Meyer, Ermonshina, 2013 ; Gregg, 2015).

Cet article consacré aux transformations de la politique taïwanaise sous l'impulsion de la démocratie électronique se concentre sur ce personnage emblématique, cette jeune programmeuse qui met ses prouesses techniques au service d'une démocratie qu'elle conçoit comme un processus toujours en marche, un travail constant dans le contexte d'une jeune démocratie taïwanaise

[3] budget.g0v.tw laccès le 21/01/2017).

[4] On parle d'open data lorsqu'une donnée numérique est diffusée de manière structurée selon une méthode et une licence ouverte garantissant son libre accès et sa réutilisation par tous, sans restriction technique, juridique ou financière.

[5] http://g0v.tw/en-US/about.html laccès le 21/01/2017). 
consolidée mais poussée à se réinventer par ses plus jeunes générations. Dans le cadre de ce numéro sur l'engagement politique et la participation des jeunes Chinois dans le monde, une enquête sur cette jeune "hackeuse civique open source » permet d'éclairer ce mouvement de démocratisation de la démocratie qui semble prendre à Taïwan, l'évolution générationnelle et politique du pays, ainsi que la «traditionnelle » appétence de Taïwan et en particulier des récents élus indépendants et du DPP pour la mobilisation des nouvelles technologies et des dispositifs participatifs. Le cas taïwanais offre un contrepoint intéressant de société qui cherche à se construire en réaction à une histoire récente autoritaire, et à une Chine non démocratique perçue comme une menace au bouillonnement démocratique. La trajectoire d'Audrey Tang incarne en un sens le besoin pour Taïwan de lutter contre les résidus autoritaires du Parti nationaliste et plus encore d'être une démocratie exemplaire pour la Chine, mais aussi pour la communauté internationale qui finira par reconnaître son existence à part entière. Cette militante de g0v est assez représentative de la volonté d'une grande partie des jeunes taïwanais d'être des citoyens actifs luttant pour que l'île soit le théâtre d'une transparence et d'une participation politique édifiantes, pour se démarquer du continent chinois et affirmer ainsi l'identité taïwanaise. Il s'agit peut-être également de le convaincre que le régime démocratique n’est pas inadapté à ses conditions et à son histoire particulières et dans le meilleur des cas, faire monter les enchères avant un rapprochement final.

Cet article, principalement issu de quatre rencontres et trois entretiens semidirectifs approfondis menés à Paris entre septembre 2015 et mars $2016^{6}$, d'une correspondance électronique régulière et de l'analyse des publications et conférences d'Audrey Tang, est une monographie cherchant à comprendre comment les dispositifs numériques de participation sont fabriqués dans le contexte spécifique taïwanais, au croisement des influences occidentales et de l'influence de son voisin chinois, et d'élucider ainsi les raisons d'une rencontre réussie entre savoir-faire technique, réflexion théorique et volonté politique.

\section{Optimisme technologique et réserves}

Le courant déterministe technologique a tendance à se concentrer sur le potentiel qu'a Internet de remédier au manque de participation et de délibération dont souffrent les démocraties dans le monde, en minimisant les obstacles à la réalisation d'un tel potentiel. Des débats intéressants et l'expression d'opinions hétérodoxes ont lieu sur la Toile et permettent d'établir un travail de surveillance et de confrontation qui est au cœur de la démocratie (Rosanvallon, 2006),

[6] Ces entretiens successifs se sont avérés indispensables pour comprendre une pensée et des actions riches et complexes, mais également pour suivre leur évolution particulièrement rapide et leur réception par les autorités taïwanaises. J'ai également eu l'occasion de rencontrer et converser avec le père d'Audrey Tang, qui l'accompagne parfois dans ses déplacements à l'étranger. 
et qu'on ne trouve pas toujours dans le débat politique classique. Les forums politiques sur Internet ont ainsi nourri les espoirs quant au progrès de la démocratie délibérative, de par leur nature décentralisée, permettant l'interaction et la participation de tous sur tous les sujets: Rheingold a ainsi déclaré que «si les forums politiques en ligne ne sont pas une technologie démocratique, c'est qu'aucune technologie ne peut l'être » (Rheingold, 1993, p. 131). L'avènement d'une « démocratie de conversation » renouvelle les interactions entre citoyens et dirigeants politiques, ce qui est censé faciliter la délibération (Jensen, 2003 ; Albrecht, 2006). Ces interactions n'ont plus seulement lieu sur des forums gouvernementaux mais sur les microblogs (Twitter et Plurk à Taïwan), où les hommes et femmes politiques communiquent désormais abondamment avec leurs concitoyens ${ }^{7}$. La société civile bénéficie également fortement des possibilités d'organisation et de délibération offertes par les outils numériques. Or les études ont montré que la délibération contribue à rendre les citoyens moins centrés sur eux-mêmes, plus enclins à la réflexion collective, à une plus grande tolérance et compréhension des positions des autres (Gutmann, Thompson, 2004 ; Warren, 1996a, 1996b). Il a aussi été démontré qu'en délibérant, les citoyens améliorent considérablement leurs compétences en matière de communication, mais également de participation, d'autonomie et d'efficacité politiques (Gastil, 1993 ; Bohman, 1996 ; Fishkin, Luskin, 1999). Cependant, Internet est perfusé par le monde social, et court par conséquent le risque de voir son potentiel démocratique d'égalité et de création d'un espace public épanoui et épanouissant court-circuité (Flichy, 2001, 2008b ; Cardon, 2010). Comme le montrent les études sur l'homophilie (Lev-On, Manin, 2006 ; Flichy, 2008a ; Fouetillou, 2008 ; Cardon et al., 2010), si Internet devient un agrégateur des intérêts individuels et le lieu d'un référendum permanent, le débat public en ressort non enrichi mais considérablement appauvri, car il n'y a pas de débat délibératif si à tout moment je clique, sans me confronter aux autres ${ }^{8}$. Jürgen Habermas affiche le même constat pessimiste face à l'émergence d'une sphère publique en ligne. La croissance des réseaux multiplie les possibilités de contacts et les échanges d'informations, sans aboutir automatiquement à l'expansion d'un monde de partage intersubjectif et à l'entrelacement discursif de conceptions et d'opinions diverses, qui sont la condition d'émergence des espaces publics politiques. Internet peut en réalité autant étendre que fragmenter les interactions et les échanges (Habermas, 1998). Il y a un risque de conformité, du fait de la possibilité de personnaliser, customiser ses sources d'information et ses plateformes d'échange et de discussion. Ce risque est devenu encore plus grand à l'heure des «filter bubbles » causées par la personnalisation des résultats sur Google, et qui nous maintiennent dans une sphère idéologiquement homogène (Pariser, 2011). Le juriste américain Cass Sunstein est l'un des pessimistes, prévenant des

[7] Plurk est plus populaire que Twitter à Taïwan (Dataopedia.com), ce qui n'a pas échappé aux candidats aux élections et élus taïwanais qui y ont un très grand nombre de followers et prétendent ainsi être mieux en prise avec leurs besoins.

[8] Les internautes tendent ainsi à camper sur leur position (Greffet, Wojcik, 2008). 
dangers de la fracture numérique, de la balkanisation des idées, de la disparition du hasard (serendipity) menant à la confrontation des expériences, des idées et des points de vue sur la Toile (Sunstein, 2001, 2007). Les études sur la démocratie Internet (Cardon, 2010), porteuse d'un espoir fort de renouvellement démocratique, concluent ainsi souvent sur une note pessimiste: la nécessaire osmose entre capacités techniques et théoriques (bonne connaissance de l'histoire et de la théorie de la démocratie, de la délibération, maîtrise de la programmation et du design, etc.) est trop rare. La volonté politique nécessaire à une institutionnalisation des innovations délibératives numériques fait cruellement défaut. Les réalisations d'Audrey Tang à Taïwan offrent par conséquent un cas d'étude particulièrement intéressant, car elle est une passeuse entre des mondes qui se connaissent et communiquent mal (Barlow, 1996) : ceux des programmeurs, des universitaires, et des décideurs. Elle bénéficie en effet de conditions politiques particulièrement favorables pour que son action porte. Son cas met en lumière une nouvelle génération de militants, pour lesquels politique et technologies sont inséparables, et elle incarne ainsi la rencontre entre la théorie politique et la compétence technologique, dont l'imbrication apparaît ainsi plus évidente qu'à l'accoutumée.

\section{Audrey Tang et l'esprit des pères fondateurs du Net ${ }^{9}$}

Audrey Tang (唐鳳) est née en 1981. Cette programmeuse de génie, jeune entrepreneure du web, a pris sa retraite à trente-trois ans pour mettre son talent au service de la politique, même si elle reste consultante pour Apple et Oxford University Press. Elle justifie son militantisme présent par son enfance bercée par l'entourage politisé de son père et bouleversée par le développement du World Wide Web. Elle a en effet grandi au sein d'exilés du mouvement étudiant de Tiananmen, dont son père fut un témoin et qui était devenu son sujet de thèse de science politique en Allemagne (université de la Sarre). Elle assiste ainsi très tôt à des débats militants, notamment sur le processus démocratique, alors que Taïwan se démocratise. Elle commence à se passionner pour la programmation à 8 ans, codant d'abord sur des cahiers et sans ordinateur, ce qui en fait pour elle une manière de penser à part entière. Son premier programme est un jeu éducatif pour son petit frère. C'est l'époque où Internet se développe et où l'informaticien britannique Tim Berners-Lee et son équipe inventent le World Wide Web. De retour à Taïwan en 1993, elle entre au collège et apprend à coder en Perl, un langage de programmation qui fonctionne grâce à la collaboration et l'entraide des programmeurs. Elle s'adapte mal au système scolaire conservateur : "J'ai abandonné l'école à 14 ans pour explorer les nouvelles possibilités offertes par le web, où je peux directement lire et même converser avec les chercheurs dont on ne m'aurait enseigné les travaux qu'à l'université, dans le

[9] La trajectoire d’Audrey Tang est reconstituée à partir de ses propos lors de notre premier entretien du 15 septembre 2015. 
meilleur des cas. $\gg^{10}$ C'est l'époque des « dotcoms » lentreprises dont les bénéfices sont principalement réalisés sur Internet), relativement peu de logiciels ont encore été développés et le web est un far west où tout reste à faire. Elle contribue à la création d'un moteur de recherche et réseau social pour les textes en mandarin, Inforian Inc., et devient entrepreneure. Avec des amis, elle crée une start-up qui utilise le langage de programmation Perl et fait grand bruit. Intel y investit. À 17 ans, elle devient consultante ${ }^{11}$.

C'est dans ces années formatrices de participation active aux premiers développements du web que la programmeuse conçoit sa vision radicalement égalitaire et participative du processus de prise de décision politique. La transparence radicale de l'information est ainsi pour Audrey Tang la première étape indispensable à une démocratisation de la prise de décision politique, et une revendication de plus en plus forte de la jeunesse taïwanaise. Elle explique que l'open data ${ }^{12}$ est une étape bien plus avancée que la liberté d'information, qu'elle compare au read only, fichier en lecture seule qu'il est impossible de modifier. Le gouvernement est sommé de révéler les informations, mais pas l'ensemble des données brutes qui servent aux prises de décisions politiques. Le point de départ de sa démarche est qu'un citoyen informé est un citoyen actif et vigilant. Une démocratie transparente est un régime dans lequel les pouvoirs publics sont réellement responsables devant leurs électeurs et les citoyens confiants à l'égard de leurs dirigeants. Avec ces données, les citoyens peuvent lancer de nouvelles initiatives à travers des mouvements comme g0v. Audrey Tang se dit profondément inspirée par l'esprit de partage, d'autogouvernement et de recherche de consensus des pionniers du World Wide Web, notamment de l'Internet Engineering Task Force, un groupe informel d'ingénieurs et de programmeurs qui a joué un rôle majeur dans la construction et le développement d'Internet ${ }^{13}$. Pour décrire cet esprit, elle explique qu'aucune institution et aucun gouvernement ne régulaient Internet à ses débuts. Il s'agissait alors d'un ensemble de protocoles conçus par des opérateurs télécoms qui s'étaient mis d'accord pour rendre disponibles leurs lignes de code sur le réseau. Tous les participants à la construction de la Toile, tous les opérateurs, ingénieurs et n'importe qui souhaitant y prendre part, devenaient simplement membres en s'inscrivant aux groupes de travail. Les documents publiés sous la forme de RFC, "Requests for Comments », donnaient lieu à de longs débats en ligne. Les participants, tous bénévoles, prenaient alors des décisions par consensus, sans système de représentation : ils soumettaient des idées à la discussion et les meilleures séduisaient un plus grand nombre d'entre eux. Quant aux idées moins bonnes, on travaillait à les rendre meilleures.

[10] Entretien du 15 septembre 2015.

[11] Elle a également lancé une plateforme de collaboration communautaire en 2000, OurInternet, Inc. ; un logiciel de traitement de texte pour les entreprises S-Team Technology en 2004 ; et Socialtext, une plateforme de collaboration sociale pour les entreprises en 2008.

[12] Mouvement d'ouverture des données administratives.

[13] Entretien du 15 septembre 2015. 
L'avantage de l'open source ${ }^{14}$, c'est qu'il s'agit d'« un espace sûr où nous pouvons apprendre les uns des autres, plutôt que d'imposer nos désirs ou volontés aux autres. Les résultats ne sont pas parfaits. Mais ce n'est pas grave parce que c'est un processus itératif, où l'on reprend constamment les choses, on repasse dessus... » (Richard, 2015). Ainsi, tant qu'il y a désaccord, la discussion se poursuit et on cherche des solutions. Quand enfin plus personne n'exprime d'oppositions, cela équivaut à un accord. Cela signifie que tous les standards techniques d'Internet sont décidés selon le principe du consensus, avec des méthodes de travail participatives et des groupes ouverts à tous. Selon Audrey Tang, c'est le professeur à MIT Dave Clark qui semble avoir le mieux rendu l'esprit de l'Internet Engineering Task Force avec sa fameuse déclaration: "We reject kings, presidents and voting, we believe in rough consensus and running code ». C'est l'idée générale, la direction globale, qui fait consensus et qui reste approximative. Ce qui importe, c'est que le code fonctionne, même si des désaccords peuvent exister lorsqu'on entre dans le détail. Contrairement au système représentatif, il n'y a pas de représentant, de chef charismatique ou encore de lobby pour prendre des décisions ${ }^{15}$. La voie choisie est celle qui fonctionne et qui fait sens pour le plus grand nombre d'acteurs.

Audrey Tang se définit comme une "anarchiste conservatrice», par exemple lors de la conférence présentant les actions de g0v organisée le 9 septembre 2015 au centre CNRS Pouchet ou sur son profil sur la plateforme de blog minimaliste Medium.com, où elle publie régulièrement depuis janvier 2016 des articles-manifestes. Ce qu'elle entend par ce pléonasme, c'est qu'elle œuvre pour la conservation de l'utopie anarchiste expérimentée par les programmeurs du Net depuis vingt ans. Il n'est pas rare que les pratiquants et connaisseurs d'un tel mode opératoire suggèrent de le transposer aux prises de décisions politiques. Le chercheur américain Clay Shirky est l'un des porte-parole les plus influents de ce courant. Dans l'une de ses conférences TED Talk intitulée «Comment Internet transformera (un jour) le gouvernement», il explique ce que « le monde de la programmation open source peut enseigner à la démocratie » (Shirky, 2012). Il passe en revue l'histoire des révolutions technologiques et la mondialisation de l'information. Il rappelle les espoirs à chaque fois déçus que l'innovation technologique en question apporte enfin la paix mondiale. Au contraire, « plus il y a d'idées en circulation, plus il y a d'idées à contredire. Plus de médias signifie toujours plus de débat. Voilà ce qui arrive lorsque l'espace des médias se développe ». Il rapproche alors le rôle révolutionnaire joué par

[14] Lorsqu'on parle d'open source, on fait référence aux logiciels qui respectent les critères établis par l'Open Source Initiative : libre redistribution, accès au code source et création de travaux dérivés. Mis à la disposition du grand public, ce code source est généralement le résultat d'une collaboration entre programmeurs. C'est précisément ce à quoi fait référence Audrey Tang lorsqu'elle utilise ce terme.

[15] C'est ce qui se passe dans l'idéal. Dans la pratique, les grandes entreprises du numérique paient des gens à plein temps pour travailler dans ces groupes afin de s'assurer que les standards soient en accord avec leurs intérêts économiques : c'est une forme de lobbying détourné. 
la revue scientifique comme moyen de synchronisation du débat dans la communauté des spécialistes des sciences naturelles au mode opératoire des programmeurs open source comme Audrey Tang. Il explique minutieusement le fonctionnement de GitHub, système de contrôle de version distribué qui a été inventé par Linus Torvalds et qui permet la collaboration sans coordination. Son message est que les « programmeurs ont mis au point une méthode collaborative à grande échelle, distribuée, bon marché et en phase avec les idéaux de la démocratie », mais que tant que la volonté politique manquera, cela n'aboutira malheureusement à aucune révolution politique.

«Les gens qui expérimentent la participation n'ont pas le pouvoir législatif et les gens qui ont le pouvoir législatif n'expérimentent pas la participation. Ils expérimentent avec l'ouverture. Il n'y a pas de démocratie digne de ce nom qui n'ait pas essayé une transparence, mais la transparence est l'ouverture dans un seul sens et se voir donner un tableau de bord sans un volant n'a jamais été la promesse de base qu'une démocratie fait à ses citoyens ».

La difficulté principale est d'intégrer ces technologies et protocoles d'échanges expérimentés et perfectionnés par les programmeurs depuis vingt ans au débat, à la vie et à l'action politique. C'est justement dans cette optique que des hackers citoyens comme Audrey Tang travaillent à rendre la délibération possible, facile et incontournable dans le contexte de la démocratie taïwanaise. Or, cela rejoint l'un des grands défis actuels de ce nouveau travail de «transition démocratique $\gg^{16}$, vers une meilleure démocratie, pour ne plus seulement se reposer sur la démocratie représentative, c'est-à-dire le vote. Il s'agit de permettre à un espace public, au sens habermassien du terme, d'émerger, où chacun peut s'informer, s'exprimer, être confronté à divers points de vue, et discuter de façon rationnelle des modalités de vie ensemble, de prendre part au processus de prise de décision par la délibération et l'usage de la raison d'une façon plus rigoureuse, régulière et moins dangereuse que les sondages d'opinion ou la démocratie directe limitée aux référendums de type Brexit (Fishkin, 2009 ; Ackerman, Fishkin, 2004).

Audrey Tang est l'une des militantes les plus actives de la réalisation de cette cause à Taïwan : elle cherche à démocratiser la politique en lui apportant l'autogestion, la collaboration, la confiance en l'intelligence collective et surtout de meilleurs outils pour rendre possibles des espaces de parole et de consensus.

[16] À la conférence de l'IPSA 2016 à Poznan, Philippe Schmitter, lors d'une commémoration des 30 ans du livre collectif qu'il avait dirigé sur la transition démocratique, reconnaissait qu'on était loin de la fin de l'histoire après la chute de l'URSS et les vagues de démocratisation successives. Il est désormais plus compliqué de convaincre de la légitimité des régimes démocratiques une fois le combat gagné et quand il n'y a plus d'alternative ou d'épouvantail à lui opposer; et surtout quand tant de limites, de frustrations et d'insatisfactions animent les sociétés démocratiques, qu'elles soient anciennes ou plus récentes. 
Sa conviction provient de l'association qu'elle a très tôt naturellement faite entre ce système virtuel et le système politique. "C'est le premier système politique dont j'ai entendu parler : tout était très organique et interactif » (citée par Richard, 2015). Les initiatives de hackers civiques se multiplient dans le monde, mais il nous semble important de faire connaître l'ampleur et le dynamisme du phénomène dans le contexte unique de Taïwan, où la pression sociale contraint le gouvernement actuel à se montrer particulièrement réceptif et à encourager la délibération en ligne.

\section{Le contexte taïwanais}

\section{Coïncidence entre démocratisation et révolution médiatique pour les jeunes générations}

La simultanéité du processus de démocratisation et du développement des médias numériques rend le cas taïwanais particulièrement fascinant. Jusqu'à la levée de la loi martiale en 1987, le Kuomintang gouverne Taïwan de façon autoritaire, même si l'idéologie officielle fondée sur les Trois principes du peuple de Sun Yat-sen présentait la démocratie constitutionnelle comme l'objectif ultime de l'État. Chen Sheue Yun a retracé l'histoire de l'interaction forte entre démocratie et nouvelles technologies à Taïwan (S. Chen, 1998). Il semble que sous la pression des jeunes générations hyperconnectées, la nouvelle présidence du DPP cherche à reprendre ce flambeau.

La population d'internautes atteignait $83,8 \%$ de la population de Taïwan en $2014^{17}$. Le réseau de télécommunications bénéficie d'infrastructures d'excellente qualité et d'un marché des opérateurs télécoms très compétitif. Dès mai 1993, un premier mouvement étudiant est organisé sur des forums de discussion; en l'occurrence le BBS de l'université nationale de Chiao-Tung, pour protester contre la mauvaise qualité de la cantine universitaire. Lors de sa conférence à la Nuit des idées publiée dans Le Monde (Tang, 2016d), Audrey Tang s'interrogeait comme elle l'avait fait lors de nos entretiens:

«Pourquoi y a-t-il à Taïwan un si grand nombre de hackeurs prêts à travailler pour la démocratie ? Je pense que cela est dû au fait que notre génération est la première à s'exprimer librement après quarante ans de dictature de Tchang Kaï-chek. 1988 a vu l'avènement de la liberté de la presse et l'arrivée des ordinateurs personnels. 1996 a été l'année de la première élection présidentielle et de l'éclosion des sites Internet. Internet et la démocratie ont évolué ensemble. »

[17] Sources : ITU et US Census bureau. 
Cette affirmation est corroborée par la thèse de Pai Chin-Fen, dans laquelle elle explique l'importance des différences générationnelles entre ses parents, qui ont vécu sous le régime autoritaire, elle-même, qui a encore des souvenirs de la loi martiale et de la répression des mouvements antigouvernement, et ces étudiants nés après la fin de la loi martiale en 1986 et qui n'ont connu ni la censure, ni le culte de la personnalité, ni la répression des mouvements sociaux. Pour eux, «parler politique est une activité sociale comme une autre. Ils n'ont pas besoin de s'inquiéter de ce qui pourrait leur arriver s'ils s'expriment en faveur du gouvernement ou protestent contre sa politique » (Pai, 2010). Le contraste est grand avec les générations précédentes, marquées par le gouvernement colonial japonais puis la loi martiale imposée par le Kuomintang, pour lesquelles les jeunes étaient incapables de parler politique, comme le reflète le dicton taïwanais: "Il vaut mieux que les enfants n'aient pas de bouche ». Par conséquent, même au cours de la phase de démocratisation de 1988 à 2000, on enseignait à ses enfants à ne jamais exprimer leurs opinions politiques en public.

\section{La place de la délibération dans la jeune démocratie taïwanaise}

Après plus de trente ans de recherches consacrées à la démocratie délibérative et dix ans d'expérimentation sur l'île, l'idée est bien implantée au sein de la société civile et des institutions, qui recourent régulièrement aux mécanismes délibératifs pour résoudre disputes et conflits. C'est à la fin des années 1980 que les premiers articles sont publiés à Taïwan pour présenter la théorie de la démocratie délibérative et envisager de l'appliquer pour résoudre les disputes environnementales. Néanmoins, c'est surtout depuis 2004 que la démocratie délibérative devient un sujet de recherche important ${ }^{18}$. En 2002, soit deux ans après la victoire du DPP aux élections présidentielles qui marque la première alternance politique de l'histoire de Taïwan, le ministère de la Santé commissionne la première série d'expérimentations de dispositifs délibératifs à Taïwan. Rapidement, différents dispositifs développés à l'étranger sont expérimentés, tels que les conférences citoyennes, les sondages délibératifs, les forums de citoyens ou les ateliers scénario. De nouveaux mécanismes délibératifs sont même inventés. Des forums sont organisés dans lesquels des citoyens tirés au sort posent des questions lors d'un débat télévisé retransmis sur les chaînes nationales aux candidats aux élections du gouverneur de Tainan en 2005 et du maire de Taipei en 2008. Ces expérimentations sont le fruit d'études académiques cherchant à combiner démocraties représentative et délibérative, dans un esprit proche de celui de Fishkin et Ackerman dans leur proposition normative d'organiser une journée de délibération avant chaque élection, tout en le

[18] Pai Chin-Fen recense 21 ouvrages consacrés à la démocratie délibérative publiés entre 2005 et 2009, et plus de dix articles et mémoires de master ou de doctorat publiés chaque année. Cela s'explique par le grand nombre de projets consacrés à la question bénéficiant de l'Agence nationale de la recherche taïwanaise (25 entre 1999 et 2012 avec un financement global de 25 millions de dollars taïwanais). 
combinant à un débat télévisé entre candidats aux élections et citoyens ordinaires (Ackerman, Fishkin, 2004). La logique de ce dispositif veut que la diffusion télévisée de l'échange rationnel et informé et le dialogue entre les citoyens et les candidats permettent aux électeurs de mieux comprendre les positions de ces derniers concernant des questions politiques majeures. Un tel dispositif est censé aider les électeurs à élire les candidats à même de répondre à leurs besoins, et pose les bases de ce qui deviendra plus tard Talk to Taiwan ${ }^{19}$.

Un bref retour sur la transition démocratique de Taïwan permet de comprendre l'assez grande appétence de l'île pour la démocratie délibérative. La transition est amorcée dans les années 1980 mais elle culmine lors de la première alternance en 2000 lorsque le DPP, profitant de divisions au sein du Parti nationaliste, gagne les élections présidentielles. Le candidat du DPP Chen Shui-bian n'est pourtant élu qu'avec $40 \%$ des voix et une minorité à l'assemblée (le Yuan législatif). Le Parti, en quête de légitimité, mobilise les mécanismes délibératifs pour se démarquer du Kuomintang et se présenter comme le champion d'une démocratie de meilleure qualité, dans un contexte de désaffection et de déceptions populaires croissantes (Huang, Hsieh, 2013). En effet, malgré la consolidation de la démocratie à Taïwan et l'alternance régulière et pacifique des deux grands partis au pouvoir en 2000, 2008 et 2016, les cas de corruption, l'inefficacité du pouvoir législatif et le manque de légitimité des institutions, des représentants élus $^{20}$ et du processus de prise de décision ont rapidement érodé la confiance et les attentes populaires vis-à-vis du nouveau régime. L'étude de l'Asian Barometer révèle un paradoxe : le soutien pour la démocratie et son fonctionnement à Taïwan est élevé, mais la confiance envers les institutions représentatives (Parlement et partis politiques) y est parmi les plus basses de toutes les démo-

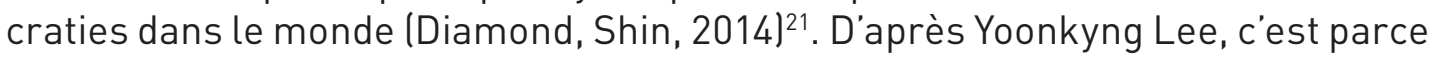
que les institutions censées représenter les intérêts des citoyens, et notamment les partis politiques, n'y sont pas suffisamment développées (Lee, 2014). De nombreux militants pensent qu'une plus grande participation des citoyens au processus de prise de décision peut permettre de remédier au manque sévère de légitimité des politiques promulguées par les élus taïwanais, qui est source d'insatisfaction populaire et de résistance à la mise en application des politiques. Une contributrice de g0v a ainsi déclaré : «Plus on retarde l'inclusion du public dans le processus de résolution des problèmes, de planification politique et dans l'agenda législatif, moins il aura confiance dans le processus politique $\gg^{22}$.

[19] Talk to Taiwan est un projet monté en parallèle de vTaiwan. Des personnalités politiques sont invitées à y répondre aux questions issues d'un forum délibératif mis en place sur Internet une semaine avant l'émission et permettant également de réagir aux réponses de l'homme ou la femme politique en direct.

[20] Les études ont montré que la méfiance envers le corps législatif a augmenté de 46 à $56 \%$ entre 1992 et 2002. Depuis cette date, elle s'est toujours maintenue au-delà de $60 \%$.

[21] C'est également le cas de la Corée.

[22] Discours en ligne de aETBlue disponible à la page suivante : https://ocf-tw.hackpad.com/ Loomio-l8fikCsKHiG laccès le 21/01/2017). 
La société civile taïwanaise est très dynamique, et de nombreux mouvements sociaux y ont régulièrement lieu, notamment pour protester contre le nucléaire. Une série de mouvements de défense de la liberté d'expression incite également la jeunesse taïwanaise à considérer le système démocratique comme la valeur centrale de Taïwan. Le mouvement des Fraises sauvages est ainsi organisé en 2008, pour protester contre la visite de Chen Yunlin, un haut cadre chinois, suivi l'année d'après par une mobilisation encore plus grande contre le monopole de la corporation Wangwang. La perspective que cette entreprise proche de Pékin ayant d'importants intérêts économiques en Chine en vienne à dominer le monde médiatique taïwanais inquiétait les étudiants mobilisés. Ces mouvements utilisent les ressources numériques pour s'organiser, communiquer et discuter et dès 2004, une expérience pilote de délibération en ligne est organisée par la société civile dans la communauté de Beitou.

D’après Audrey Tang, le « mouvement 1985 » qui a lieu en août 2013 est une étape importante de politisation de la jeunesse et de renouveau technologique des mobilisations sociales à Taïwan ${ }^{23}$. La vingtaine d'organisateurs du mouvement réclamant plus de transparence sur la mort d'un jeune soldat, Hung Chung-chiu, parvient en effet à se coordonner en ligne et à crowdsourcer l'organisation d'une marche de 250000 personnes. Après le succès de leur mouvement, ils publient le résultat du crowdsourcing (ou production participative), une sorte de manuel pour débutants de mouvements civiques sous la forme d'un document Google. Le document est en open source, de sorte que des militants plus expérimentés y contribuent. On y débat des avantages et inconvénients d'un prototype de diffusion et de retransmission des manifestations sur Internet.

\section{Le mouvement des Tournesols}

L'aboutissement de cette politisation de la jeunesse taïwanaise est un mouvement d'occupation de grande ampleur, né dans le sillage des mouvements 15- $M$ en Espagne et Occupy Wall Street de 2011. Il a bénéficié d'une large couverture médiatique internationale. Au printemps 2014, l'hémicycle du Parlement taïwanais est occupé pendant 24 jours par 200 étudiants, alors que des milliers d'autres campent jour et nuit à ses abords. Le mouvement est largement soutenu par la population : le 25 mars, 500000 personnes descendent dans la rue, un tournesol à la main. Ce qui a déclenché le mouvement étudiant dit des Tournesols lqui représentent la transparence et l'espoir), c'est la procédure expéditive de ratification d'un accord signé avec la Chine le 21 juin 2013 et portant sur le commerce des services (Chuang, 2016 ; Hsiao, Wan, 2016). Du point de vue d'Audrey Tang, les manifestants ne souhaitent nullement prendre le pouvoir, ni que le Parlement soit dissous et qu'il y ait de nouvelles élections, mais simplement que les élus respectent des procédures démocratiques. La très forte présence du mouvement sur les réseaux sociaux est

[23] Entretien du 15 septembre 2015. 
l'une des particularités de cette mobilisation (Lin, 2014), notamment grâce à la logistique fournie par la programmeuse et ses partenaires de g0v.

Dix jours avant le début du mouvement des Tournesols, dans le cadre d'un défilé de protestation contre la construction d'une centrale nucléaire, Audrey Tang et g0v collaboraient avec un autre groupe de militants spécialisés dans la retransmission open source de conférences en ligne, et diffusaient en direct l'intégralité de la marche et des discours à une audience qui ne s'était pas déplacée sous la pluie mais était exceptionnellement large sur Internet. Les programmeurs peuvent ainsi réagir rapidement lorsque le Parlement est soudainement occupé quelques jours plus tard. C'est ce que Clay Shirky a appelé « l'application situationnelle » : g0v installe cinq caméras dans le Parlement et un grand écran sur sa façade. Les débats, mais également les moindres faits et gestes des occupants du Parlement, sont diffusés en ligne et à l'extérieur du bâtiment. Le mouvement est un moment fondateur pour Audrey Tang, même si elle déclare ne pas y être directement engagée, au sens où il n'est pas question pour elle de prendre position mais d'œuvrer sur le plan technologique pour qu'il soit marqué du sceau des valeurs des pères fondateurs du Net. En tant que « hackeuse civique open source », son rôle est de créer et de mettre à la disposition des manifestants des outils facilitant transparence, libération de la parole et délibération. Elle contribue à la création du site Internet où leurs discussions sont diffusées en direct, enregistrées et archivées, à l'aide d'un système de transcriptions crowdsourcées. Résultat : "C'était le “Truman Show" ! Tout ce qui s'y passait était une pure performance, rituelle et très consciente d'elle-même. Les vrais débats, la vraie action, ça se passait dans les rues autour du Parlement » (Richard, 2015). Audrey Tang explique que dans chacune des trois rues qui encerclent le bâtiment s'étaient regroupés respectivement des séparatistes, des militants de gauche, et des écologistes. Toutes sortes de débats et délibérations entre petits groupes ont lieu, et leur diffusion en direct en ligne et leur transcription les rend accessibles à des centaines de milliers de personnes. La transparence des débats encourage le pacifisme, et on assiste rapidement à la « pollinisation croisée » des occupants, qui n'hésitent plus à quitter leur groupe de rattachement (pol.is). La programmeuse décrit avec fierté comment les technologies ainsi mises à disposition des manifestants favorisent le consensus issu de la rencontre physique d'acteurs animés par différentes idéologies mais que les circonstances rassemblent, ainsi que la possibilité d'expansion des bonnes idées grâce aux technologies mobilisées. Concrètement, à l'issue de la mobilisation, les étudiants s'entendent principalement sur la nécessité d'étendre ce processus délibératif partout.

En ce qui concerne les programmeurs de g0v, ces trois semaines de participation intense loù ils sont constamment appelés à coder pour répondre aux nouveaux besoins et à adapter - quand c'est pertinent - les outils et pratiques de leurs actions précédentes), permettent au groupe de gagner en ampleur et en efficacité. Le mouvement Occupy Central légalement appelé mouvement des Parapluies) à Hong Kong reprend d'ailleurs quelques semaines plus tard les principes et la logistique de retransmission intégrale en ligne des faits et 
gestes des manifestants. L'autre impact de cette présence constante des caméras est la remarquable discipline dont font preuve les Occupants à Taïwan puis à Hong Kong. Ces retransmissions empêchent les débordements, en partie parce qu'elles rappellent constamment aux manifestants qu'ils sont en train d'écrire l'histoire, avec leurs moindres faits et gestes et paroles diffusés, qui prennent ainsi une ampleur bien plus large. «Nous ne sommes pas seulement en quête de concessions et de bénéfices matériels. Nous sommes en quête non d'un idéal mais d'une meilleure démocratie que celle pratiquée jusqu'ici. » ${ }^{24}$ Pratique et théorie ne sont pas séparées selon Audrey Tang, qui décrit le «processus itératif » de production théorique sur le terrain.

"Nous n'avons prêché, formé ou orienté les actions de personne mais des théories sont rapidement apparues sur les réseaux sociaux et dans la presse pendant le mouvement d'occupation. Tous les jours, de nouveaux discours y étaient produits [...] et tous les matins, les étudiants lisaient les médias au lever : la presse grand public et une sélection d'auteurs et de militants publiant du Parlement occupé leur analyse des actions en cours. C'était un processus itératif. Chaque jour, nous revenions sur la journée précédente et nous demandions ce qui avait été fait différemment et quel effet cela avait. Ce cycle itératif ininterrompu a rendu les étudiants conscients de leurs actes ou du moins de ce que les autres pensent qu'ils font. $»^{25}$

Pour Audrey Tang, qui a traduit en partie Networks of Outrage and Hope (Castells, 2012) et se réfère volontiers à l'ouvrage de Castells, c'est la théorisation sur le terrain des mouvements Occupy et de leur utilisation des nouvelles technologies qui est la plus utile à leurs participants. Les demandes d'approfondissement ou de démocratisation de la démocratie émises et théorisées lors du mouvement ont en effet beaucoup plus de prise sur les jeunes taïwanais, et rendent la théorie délibérative beaucoup plus attractive et populaire. L'impact est non négligeable sur les élections qui ont lieu six mois plus tard, et les programmeurs comme Audrey Tang s'évertuent à maintenir la flamme allumée en concevant des outils technologiques qui facilitent et amplifient l'expression et la participation citoyennes auxquelles la jeunesse taïwanaise a goûté et ne veut plus renoncer.

\section{Changement de style au sommet : open data et collaboration avec la société civile}

La mobilisation politique d'une partie de la jeunesse taïwanaise est une lame de fond déjà lancée avant le mouvement, mais celui-ci lui donne une accélération

[24] Entretien du 15 septembre 2015.

[25] Entretien du 15 septembre 2015. 
remarquable. De plus, l'utilisation de nouvelles technologies et de réseaux sociaux de plus en plus sophistiqués et adaptés aide à sortir de l'apathie et à accéder à moindre coût à une meilleure qualité de la participation et de la représentation (Tso, 2014). Le mouvement des Tournesols, pendant lequel ces nouveaux outils et procédures sont testés et analysés par un large public, est par conséquent un moment fondateur. Tang explique: «Le paysage politique a changé après ça. Les gens ont commencé à demander que les décisions politiques soient le fruit d'une démocratie délibérative, et pas seulement des représentants élus » (citée par Richard, 2015). De nouveaux profils émergent des élections locales de novembre 2014, qui marquent une profonde défaite du Kuomintang. Des positions clés sont désormais occupées par des hommes et des femmes politiques ne faisant campagne ni au sein du Kuomintang ni du DPP. Le nouveau maire de Taipei Ko Wen-je est un médecin qui a fait campagne comme indépendant. Suite à la démission du Premier ministre, c'est Ma Chi-Kuo, un ancien ingénieur, qui accède aux fonctions de Premier ministre de Taïwan. Le vice-premier ministre est lui aussi un ingénieur qui a travaillé pour Google, Simon Jung. Cet indépendant est depuis devenu Premier ministre. C'est encore une avancée formidable pour g0v, les velléités de transformation de la bureaucratie et du gouvernement rencontrent une écoute officielle inédite. «Cette nouvelle génération politique parle le langage des "Occupiers". Ils nous ont dit : "Vous avez dit que vous pouviez faire mieux que les législateurs. Prouvez-le maintenant." Et c'est ce qu'on fait depuis » (Richard, 2015).

La transparence radicale de l'information est l'un des grands principes défendus par les pionniers du web et les programmeurs engagés depuis. C'est la première étape indispensable à une démocratisation de la prise de décision politique et une revendication de plus en plus forte de la jeunesse taïwanaise, qui semble être mieux entendue depuis le mouvement des Tournesols. Une démocratie transparente est un régime dans lequel les pouvoirs publics sont réellement responsables devant leurs électeurs et les citoyens confiants à l'égard de leurs dirigeants. Avec ces données, les citoyens peuvent lancer de nouvelles initiatives à travers des mouvements comme g0v, qui cherchent à rendre ses données compréhensibles à travers une infographie accessible. Le maire de Taipei, Ko Wen-je, a ainsi déclaré que lorsque l'open data sera devenu quelque chose de familier pour le grand public et que les citoyens auront accès aux mêmes informations que les fonctionnaires, ils pourront faire des propositions intéressantes. « Le budget participatif faisait partie de mes promesses de campagne. Il ne suffit pourtant pas de rendre le budget d'une ville public. Il faut également que les citadins comprennent parfaitement le budget. C'est seulement alors qu'ils peuvent participer. »

Le design des plateformes de délibération, tout comme la conception des dispositifs délibératifs hors ligne ${ }^{26}$, est absolument crucial pour la réussite et la légitimité de l'entreprise. L'importance du design est certainement encore supérieure dans les délibérations en ligne, car le risque de perpétuer des inégalités

[26] Rappelons que James Fishkin a ainsi breveté le protocole à suivre dans les sondages délibératifs. 
socio-économiques, éducatives, de charisme ou de genre, notamment dans les prises de parole, mais également de perdre des participants du fait de la complexité technique de la plateforme, est grand (Badouard, 2014 ; Wright, Street, 2007 ; Monnoyer-Smith, 2006). Rien ne peut être laissé au hasard. Au-delà des contingences contextuelles, des choix techniques mais aussi politiques sont faits lorsque les forums de discussion et plateformes de délibération sont conçus, et leur design a un impact réel sur la qualité des discussions et des interactions entre participants (Pai, 2010).

Le fait que les sites gouvernementaux détournés par g0v soient finalement officiellement reconnus d'utilité publique, au sens où leur design rend les informations publiées nettement plus faciles d'accès, n'est pas anodin. Cela marque une rupture majeure de la part des autorités, qui lâchent ainsi prise. Sur le site «forké ${ }^{27}$ du Parlement, ly.g0v.tw, la place des PDF et documents Word, la description et l'origine des projets de loi sont représentées de façon ludique et lisible, et tous les amendements apparaissent clairement. Pour favoriser les débats, chaque section peut être enregistrée en signet et postée sur les réseaux sociaux. Le paragraphe en question devient ainsi un objet de discussion. Le budget est présenté de façon similaire sur budget.g0v.tw.

Figure 1. Version proposée par g0v du budget gouvernemental sur le site officiel « forké »
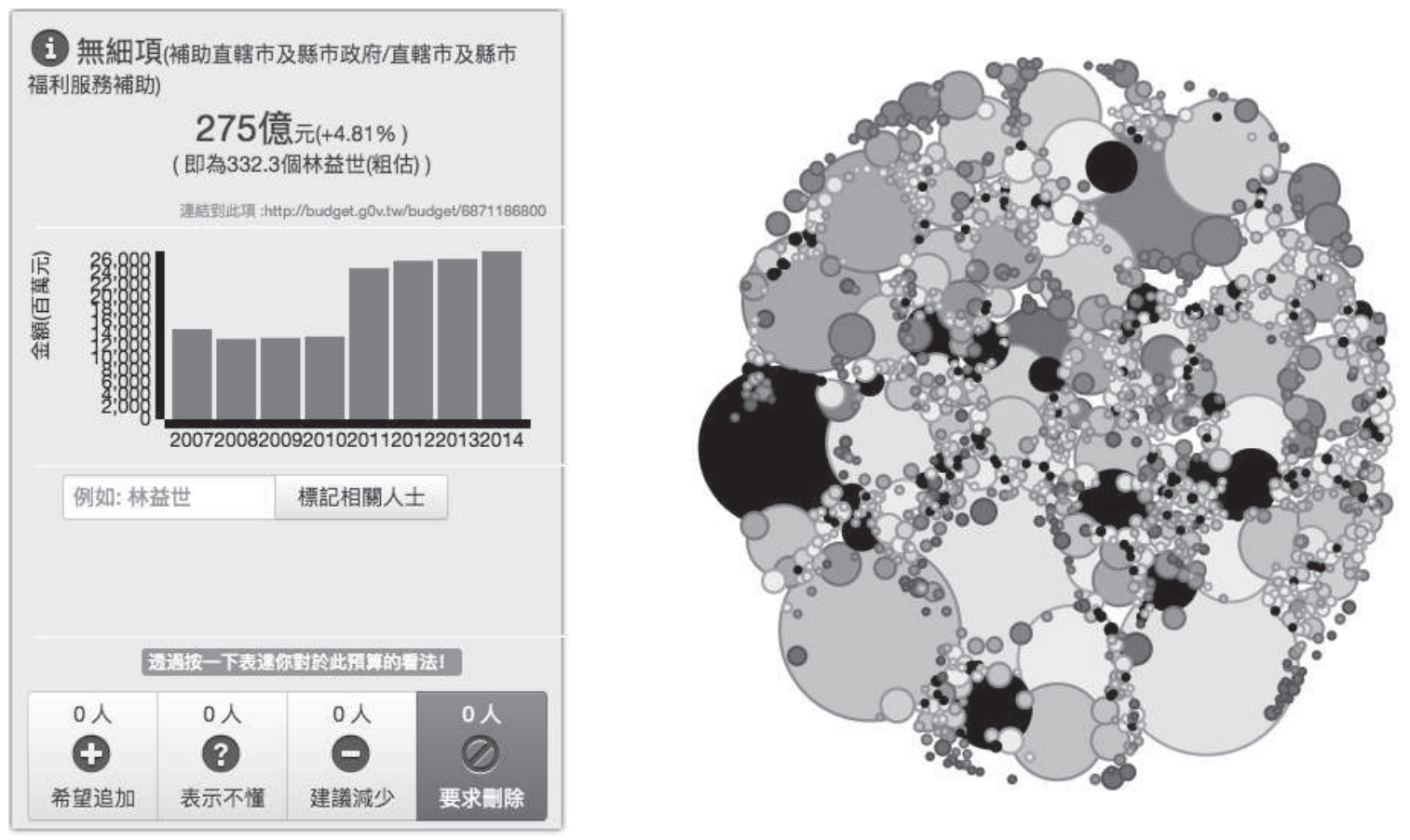

[27] Il s'agit de la bifurcation, ou la page web alternative mise en place par g0v : ly.g0v.tw au lieu de ly.gov.tw. 
La mairie de Taipei a repris cette technologie en septembre 2015, en collaboration avec g0v : budget.taipei. De nombreuses villes dans le monde rendent déjà leur budget transparent et compréhensible de tous grâce aux nouvelles technologies, mais les programmeurs taïwanais ont innové en facilitant l'interactivité et la discussion autour de chaque section, et le site a rapidement attiré plus d'un million de visiteurs.

La passation de pouvoir de janvier à mai 2016 confirme le passage radical à l'open data du gouvernement taïwanais. Le style de la présidente et du Premier ministre se veut différent, moins agressif envers l'administration précédente, à laquelle appartenait déjà Simon Jung. Il avait alors déjà accepté le principe de l'open data au sein des ministères, et lancé l'analyse et la sélection systématiques des données pouvant être ouvertes et celles devant rester secrètes. Taïwan est ainsi devenue pionnière de l'open data gouvernemental, classé première par le Global Open Data Index pour l'année 2015 devant la Grande-Bretagne et le Danemark (la France est en dixième position), et deuxième en $2016^{28}$. Les quatre mois de transition n'ont pas été passés aux revanches partisanes et à l'effacement des données pour y interdire l'accès au nouveau gouvernement, mais plutôt à leur transfert de façon transparente. L'intérêt de ce transfert est encore plus grand au sens où il a été accordé à l'ensemble de la population taïwanaise, révélant de façon inédite comment le gouvernement opère. Pour Audrey Tang, cela indique qu'« on ne passe plus d'un suzerain à un autre mais d'un suzerain à une dissémination plus démocratique de l'information, qui donne à la population accès aux mêmes données qu'aux ministres $»^{29}$. «Avec la politique nationale de l'“open data par défaut", obtenir que des informations brutes soient publiées n'est pas le problème. C'est plutôt les transformer en objets sociaux permettant la discussion et la contribution de tous » (Tang, 2016c).

\section{Le cœur de l'action de g0v}

Audrey Tang corrobore l'affirmation de Larry Wall selon laquelle la paresse est la première vertu du programmeur, au sens où il tente de raffiner les outils et protocoles à sa disposition, et de travailler à l'automatisation des tâches pour essayer de gagner du temps plutôt que de les accomplir avec application (Wall, 1996). La paresse est par conséquent une vertu dans le milieu de la programmation car c'est le moteur du perfectionnement et de l'innovation. Les programmeurs sont ainsi bien placés pour comprendre la paresse des jeunes qui ont peu de temps à consacrer aux questions politiques. Audrey Tang et g0v, contrairement à Morozov (2009), ne jettent pas la pierre aux clicktivistes. Certes, Facebook et autres réseaux sociaux donnent l'impression aux internautes de participer, d'être actifs politiquement alors que l'impact de leurs clics est extrêmement

[28] https://index.okfn.org/place/tw/ et http://index.okfn.org/place/ laccès le 21/01/2017).

[29] Entretien du 25 janvier 2016. 
réduit. Le clic, le like ou le partage sur les réseaux sociaux sont néanmoins cruciaux pour la «gratification immédiate » que ces cinq ou dix secondes donnent aux internautes ${ }^{30}$. G0v cherche ainsi à mettre à profit la satisfaction produite par des actions insignifiantes en ligne pour transformer la politique taïwanaise. Le groupe se donne pour mission de créer des moyens de mobiliser ces internautes paresseux et de transformer leurs clics en actions concrètes et efficaces. Ce sont des adeptes du microtasking, dont les études sur le crowdsourcing ont confirmé l'efficacité (Kittur, Chi, Suh, 2008 ; Rothwell et al., 2016). Il s'agit d'utiliser le « surplus cognitif »- les quelques secondes nécessaires à l'écriture d'une légende sur une photo de chat pour qu'elle soit partagée de façon virale sur la Toile - pour accomplir une mission bien plus importante sans que cela coûte davantage à l'internaute, s'il ne le souhaite pas, ou bien de s'engager davantage et de monter dans l'échelle de la participation en ligne théorisée par g0v : du clic au commentaire, du partage d'opinion à l'analyse, à la délibération et enfin à l'hacktivisme, c'est-à-dire à l'agenda setting, la définition des priorités. "Chaque étape compte et évite que le passage du clic à l'hacktivisme ne devienne une falaise infranchissable. » Audrey Tang a produit un visuel de cette ascension vertueuse de la participation en ligne et la présente en conférence sous cette forme :

Figure 2. Graphique présenté par Audrey Tang lors d'un séminaire à Paris le 9 septembre 2015

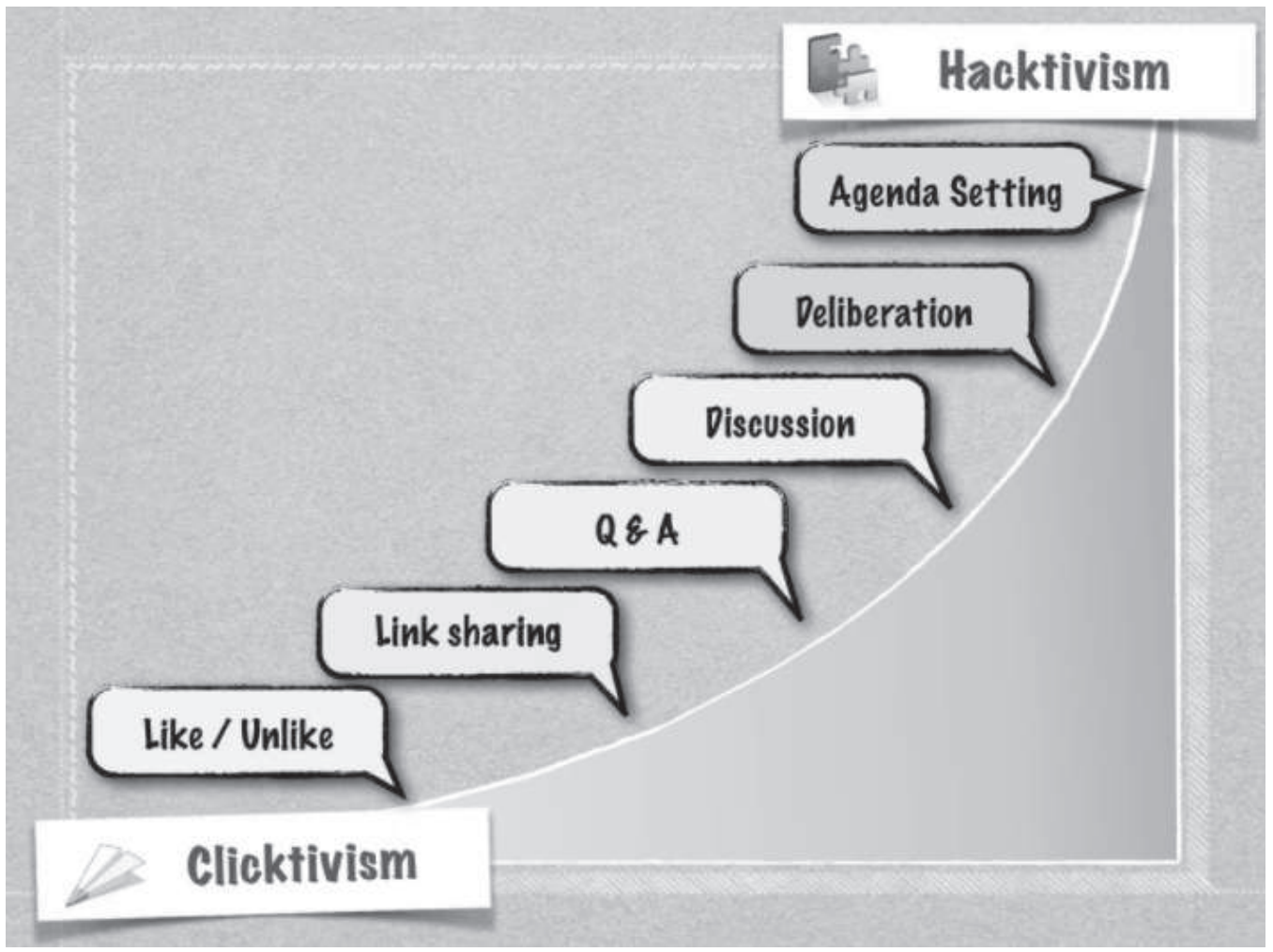

[30] Le clicktivisme permet également de décloisonner la participation en s'adressant à des publics qui ne maîtrisent pas bien l'écrit (Badouard, 2016). 
Deux projets phares auxquels a contribué Audrey Tang peuvent nous permettre de mieux comprendre comment elle réussit à orienter et agréger le surplus cognitif des internautes les plus paresseux ou occupés vers des projets ambitieux, et ainsi créer un cercle vertueux d'engagement et de participation.

Dans l'esprit d'un projet qu'elle lance en 1995, Moedict, de numérisation d'un dictionnaire chinois-chinois par crowdsourcing, g0v a demandé aux internautes de contribuer à un vaste projet de publication en ligne des finances de campagne des législateurs taïwanais. Celles-ci sont en effet uniquement disponibles en format papier dans les locaux de l'Yuan de contrôle, l'organe de surveillance, notamment de la corruption des fonctionnaires. Plus de 10000 contributeurs répondent à l'appel et acceptent de donner quelques secondes de leur temps pour faire de la reconnaissance optique de caractères ${ }^{31}$; l'ensemble de la documentation, soit 2637 pages, est ainsi numérisée et téléchargée en 24 heures. Le processus est rendu ludique par le design du site Internet, où une barre de progression donne du sens et une gratification immédiate à leur contribution. La numérisation de ces comptes de campagne a un impact important sur la politique taïwanaise. Elle rend notamment possible un second projet, le guide g0v des élections législatives (http://vote.ly.g0v.tw/). Il s'agit d'un site Internet où les informations permettant de mieux connaître chaque candidat aux élections (revenus, conflits d'intérêts possibles, actions et prises de parole au Parlement, etc.) sont présentées de façon lisible, transparente et interactive pour que les électeurs se décident de façon informée et rationnelle. Un simulateur de votes est également proposé pour éviter les votes inutiles. Dans le système électoral hybride taïwanais, qui donne à chaque électeur deux voix aux élections législatives lune pour un parti et l'autre pour un candidat locall, c'est un outil essentiel car les électeurs ont du mal à anticiper qui sera élu grâce à leur vote. À Taïwan, les voix des électeurs sont effet en partie distribuées par les partis, et les partis récoltant moins de $5 \%$ des voix n'obtiennent aucun siège à l'Assemblée. De plus, la Constitution garantit $33 \%$ de sièges réservés aux femmes, ce qui complique encore les prédictions. Le simulateur en ligne est ainsi conçu par g0v pour permettre aux électeurs de s'assurer que leur vote ne sera pas perdu ${ }^{32}$. Dans leur première édition, le guide et le simulateur sont uniquement alimentés par la société civile. Mais leur réussite est telle que le Comité électoral (CEC) prend contact avec les hackers de g0v et rend l'intégralité de leurs données accessibles en open source, si bien que les électeurs ont depuis accès au profil complet des candidats aux élections avec au moins deux semaines d'avance sur le guide des élections législatives, alors que les informations étaient auparavant disponibles en version papier trois jours seulement avant les élections. Et si les candidats décident toujours de modifier leurs données au dernier moment, ce qui arrive fréquemment, cela ne pose guère de problème en version numérique.

[31] Cela consiste à reconnaître des chiffres ou des lettres scannées et à les retranscrire dans un format qui permet de les réutiliser.

[32] Entretien du 25 janvier 2016. 
Il est important de comprendre ici le positionnement de g0v. Le groupe de programmeurs défend la neutralité du Net et a pour objectif d'approfondir l'expérience démocratique par le biais de la création d'outils technologiques qui se veulent «neutres ». Cette recherche de neutralité n'est pourtant pas dépourvue de normativité ni ne constitue un frein à l'action. Elle est au contraire inséparable de la défense d'une transparence radicale et justifie les efforts de rééquilibrage des asymétries informationnelles.

«En théorie, les autorités, les politiques et les législateurs fournissent un grand nombre d'informations aux citoyens sous forme de régulations, de mises en application, etc. Mais tous les quatre ans, nous autres citoyens n'exprimons qu'une poignée d'informations le jour des élections, en choisissant un candidat parmi seize. L'asymétrie entre le téléchargement et la mise en ligne ldownload and upload), pour ainsi dire, est très grande. C'est la raison pour laquelle il nous semble important que ce que savent les législateurs et les partis soit rendu accessible au grand public afin de lui donner les moyens de vérifier leurs déclarations et promesses. Lorsqu'un législateur prétend avoir toujours voté contre la ligne de son parti, nous pouvons consulter les archives parlementaires et affirmer que ce n'est en réalité pas le cas. C'est essentiel pour décourager le bluff et les déclarations contrefactuelles. Vouloir être neutre signifie dans notre cas que le flux d'informations ne devrait pas être unidirectionnel mais au moins bidirectionnel et être accessible de tous. $»^{33}$

La neutralité d'Audrey Tang et ses acolytes comprend d'autre part un volet décisionnel de recours à la délibération, processus visant à recueillir les souhaits des citoyens et à les fondre ensemble jusqu'à l'obtention d'une décision cohérente.

" Il s'agit de fondre les désirs, pensées et désirs supposés des gens comme dans un blender. Si l'on place trop de fruits différents dans un blender, le mélange peut prendre feu à cause des frictions et des frontières chaudes entre ces fruits. C'est la raison pour laquelle il nous faut ajouter suffisamment d'eau, la neutralité, une plateforme neutre et sûre qui interagit avec tout sans s'immiscer nulle part. Nous discutons également de cas très spécifiques, ce qui correspond à couper la pomme en tranches très fines parce qu'elles se mélangent mieux. Au lieu de débattre de la Constitution dans son intégralité, nous sélectionnons un aspect pour en délibérer, pour une plus grande cohérence des participants à la délibération et pour un mélange plus facile à obtenir. $»^{34}$

[33] Entretien du 15 septembre 2015.

[34] Entretien du 25 janvier 2016. 


\section{La technologie peut-elle sauver la délibération?}

Suite au mouvement des Tournesols et sous l'impulsion d'une initiative lancée à l'université Cornell, e-rulemaking, Audrey Tang participe à la création d'une plateforme de démocratie participative permettant aux citoyens de débattre et d'initier des projets de loi sur des sujets les concernant, dans un premier temps des questions technologiques. Elle est également en charge de leur modération et de leur fluidité. La plateforme d'e-pétition join.gov.tw est rapidement étendue à d'autres domaines, et la première loi initiée sur cette plateforme concerne la régulation de l'immunothérapie eontre-te-eaneer. C'est un patient contraint de faire traiter son cancer au Japon qui lance l'initiative de cette nouvelle régulation. La proposition de « rendre l'immunothérapie contre le cancer disponible aux patients taïwanais et d'accélérer l'utilisation de nouveaux traitements contre le cancer» obtient 5548 signatures de soutien. Dès que le seuil des 5000 signatures est atteint, des médiateurs externes et internes collaborent pour demander des clarifications, publier les données nécessaires, assurer la liaison avec et entre les différentes institutions concernées et fournir des réponses spécifiques. Une rencontre du patient à l'origine de l'initiative citoyenne est organisée au ministère de la Santé pour clarifier ses demandes et un compte-rendu est rendu public. Le mois suivant, les informations pertinentes à la question sont publiées et deux mois jour pour jour après le lancement de l'initiative, les réponses officielles sont publiées. Tout au long du processus, les débats sont filmés, retransmis et retranscrits et les rencontres avec les experts, associations et militants intégralement transcrites et publiées en ligne, pour offrir la possibilité aux citoyens intéressés de se faire leur opinion et de voir comment le ministère de la Santé a finalement pris sa décision. Cette transparence radicale a deux ambitions : « Nous ne voulons pas seulement une technologie ouverte et délibérative. Nous voulons également éclairer les décisions prises à portes closes. $»^{35}$ Leurs souhaits pourraient être exaucés avec l'équipe dirigeante actuelle.

Si les dispositifs délibératifs ont pourtant globalement moins le vent en poupe ces derniers temps, c'est principalement pour deux raisons : ils sont difficiles à organiser et ont peu d'impact sur les politiques publiques (Blondiaux, Fourniau, 2011) ${ }^{36}$. Les initiatives de la communauté open source sont relativement

[35] Entretien du 21 mars 2016.

[36] Yves Sintomer cite à ce propos la métaphore proposée par John Parkinson pour différencier les processus participatifs:

«Ils peuvent impliquer des conséquences sur le "housing", c'est-à-dire les questions structurelles; sur le «building», à savoir des problèmes importants mais de seconds plans; ou enfin se focaliser sur le «painting », c'est-à-dire sur des enjeux en aval et relativement marginaux ». Très souvent, les discussions sur le painting sont conçues pour faire oublier le plus important; et les hommes politiques pratiquent une écoute sélective (Sintomer, 2011, p. 236).

Un cas pourtant intéressant d'institutionnalisation au niveau régional est celui de la Toscane (Lewanski, 2013). 
nombreuses ${ }^{37}$ mais l'écoute dont celles de g0v bénéficient et leur impact sur les décisions du gouvernement taïwanais sont rares, comme le remarque Liz Barry :

"J'ai découvert que depuis le mouvement des Tournesols, les membres de la communauté open source et le gouvernement taïwanais collaborent pour développer une association inédite et efficace de technologies civiques, engagements gouvernementaux et médias de masse destinés à étancher la soif de discussion publique d'une nation qui se démocratise. Ils l'appellent vTaiwan. Ce que Taïwan [...] est sur le point de créer est sans précédent. Si la règle, comme l'expérience nous l'a si implacablement montré, veut que tout le code écrit pour la démocratie délibérative ne parvienne jamais à trouver formellement sa place dans un gouvernement, voici enfin un exemple qui remet en cause cette règle » (Barry, 2016).

vTaiwan est en effet l'aboutissement remarquable de plusieurs années de maturation théorique et pratique pour $\mathrm{g} 0 \mathrm{v}$, une synthèse magistrale de leurs divers projets et des outils numériques de délibération créés à travers le monde, comme Loomio, airesis, occupy.here ou pol.is (Frenkiel, 2016). Le facteur déterminant de sa réussite est avant tout la coopération des programmeurs, Audrey Tang en tête, avec des membres du gouvernement dont principalement la ministre Jaclyn Tsai. Cette ancienne avocate qui travaillait pour IBM a intégré le gouvernement en tant que ministre sans portefeuille pour se charger de la politique des nouvelles technologies ainsi que de la coordination interministérielle, le poste qu'Audrey Tang est finalement appelée à reprendre à partir du $1^{\text {er }}$ octobre 2016. En décembre 2014, elle lance un défi de taille à g0v lors d'un hackathon ${ }^{38}$ : si les programmeurs parviennent à créer une plateforme de délibération rationnelle sur des questions politiques à laquelle l'ensemble de la population peut potentiellement participer (carte blanche leur est donnée sur la conception, la régulation et la modération), le gouvernement s'engage formellement à prendre en compte les résultats de la consultation et la volonté populaire ainsi exprimée dans le processus de décision politique.

Il faut entendre ou lire la manière dont Jaclyn Tsai explique la genèse et le fonctionnement de vTaiwan lors de son interview à Talk to Taiwan, un talk-show délibératif expérimentant avec la réalité virtuelle, pour comprendre la radicalité du changement de style gouvernemental. La ministre décrit vTaiwan comme une expérience née du chaos qui a suivi le mouvement des Tournesols, ainsi que de son agacement devant la lenteur du temps politique (3 à 5 ans pour l'avancée d'une politique publique) par rapport au temps technologique.

[37] En témoigne le développement exponentiel de la civic tech.

[38] Pour une présentation intéressante des hackathons, voir Gregg (2015), et Meyer et Ermoshina (2013). 
«Il est presque certain que des voix différentes s'élèvent concernant chaque question politique. Mais avec une telle diversité de points de vue, comment atteindre efficacement toutes les parties concernées et comment parvenir rapidement à un consensus? Nous avons besoin d'un mécanisme pour cela. Je me suis donc rendue au hackathon de gOv et ai proposé ce projet. J'ai expliqué que je travaillais sur des projets de loi et que nous avions besoin d'une plateforme pour permettre à l'ensemble de la société taïwanaise de s'engager dans une discussion rationnelle à leur propos. Audrey Tang et beaucoup de bénévoles ont heureusement trouvé que l'idée était intéressante et il ne leur a fallu que quelques semaines pour créer cette plateforme [...] Nous nous étions mis d'accord pour que les procédures des discussions soient établies et maintenues par les bénévoles de g0v, qui définissent les règles du jeu et développent tout cela. En ce qui concerne le gouvernement, je me suis assurée qu'une fois une question posée, les ministères concernés répondent avant une semaine. Si un consensus est atteint en ligne, la question est réglée. Sinon, nous organisons des réunions de consultation en streaming en direct, auxquelles sont conviés les ministères et commissions, représentants du gouvernement et chercheurs concernés, et autres représentants des entreprises et de la communauté des internautes. L'intégralité de la réunion est retransmise en ligne en direct. Tout le monde exprime son opinion et tout est enregistré, ouvert et transparent. Les collègues de gov ont créé une infrastructure qui permet de mettre en ligne une transcription sténographiée deux ou trois heures plus tard. Avec cette plateforme, nous avons travaillé sur la loi sur les sociétés fermées, le crowdfunding, la vente de matériel médical sur Internet, etc. On y a délibéré de toutes ces questions, et les différents points de vue ont été enregistrés.

Quel est l'apport pour nous autres hommes et femmes politiques? Pour chaque politique, je mets en ligne toutes les informations dont je dispose sur la question de sorte que vous puissiez voir les mêmes données que moi si vous voulez vous consacrer à la question. Quand tout le monde est logé à la même enseigne, une vraie discussion peut commencer. Sinon, le dialogue reste trop vague. Si nous prenons tous le temps de comprendre le problème, de consulter les données et d'écouter les opinions des autres avant d'entrer dans la discussion, nous avons beaucoup plus de chances d'atteindre un consensus. $»^{39}$

Cette citation montre à quel point la ministre et les militants de g0v partagent le même idéal de gouvernement transparent, de citoyens informés et actifs et de délibération pour atteindre un consensus ${ }^{40}$. vTaiwan et Talk to Taiwan fonctionnent de pair : pour lancer chaque discussion, une enquête pol.is est distribuée

[39] https://www.youtube.com/watch?v=digOWjnD3pQ\&t=10m (accès le 21/01/2017).

[40] Dans ce contexte, il semble moins extraordinaire que la jeune hackeuse ait été nommée pour reprendre les missions de Jacklyn Tsai. 
Figure 3. Délibération sur vTaïwan au sujet de la régulation d’Uber
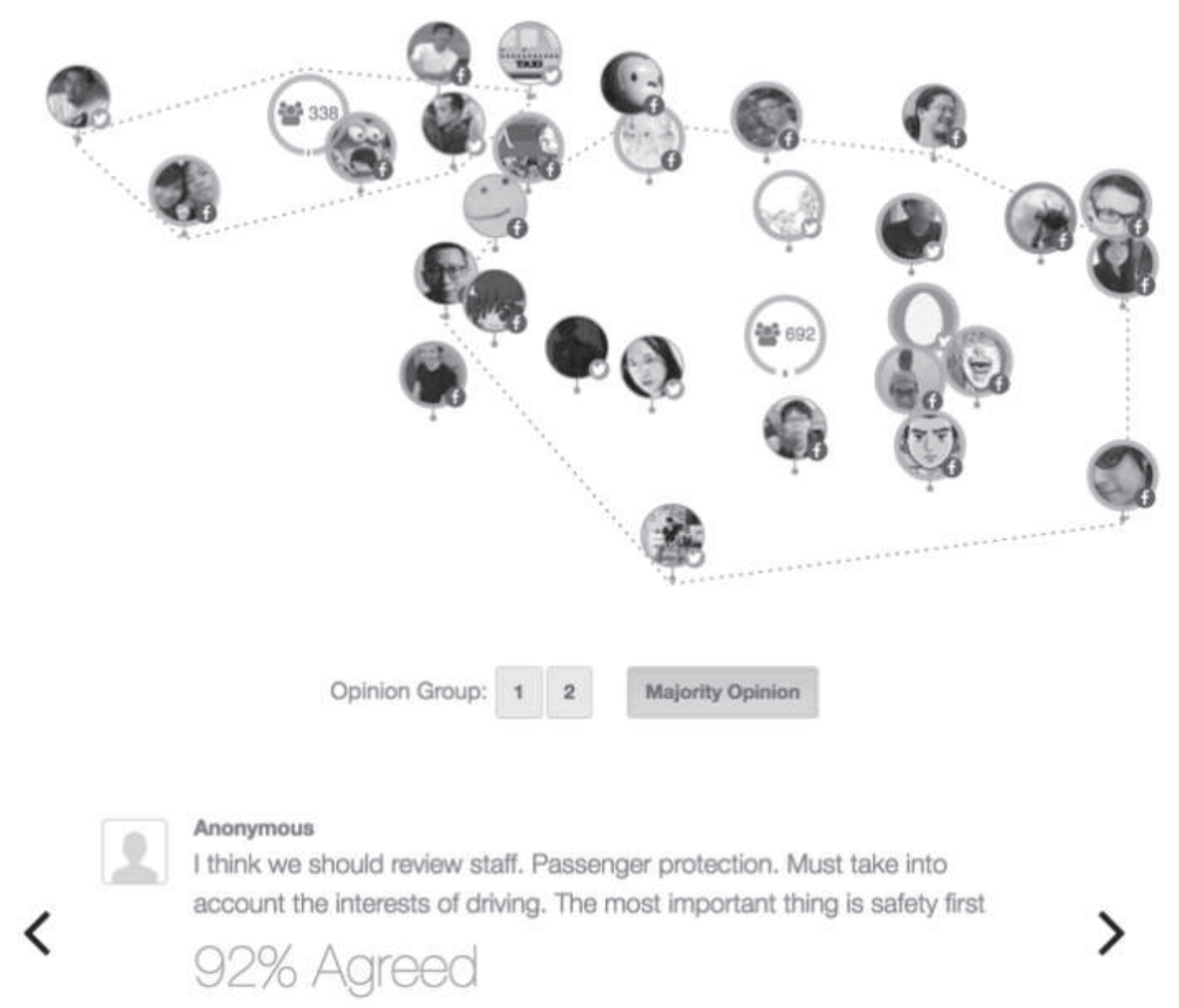

à 2000 personnes sur la page Facebook de Talk to Taiwan. Les résultats de l'enquête servent de base à des délibérations diffusées en direct, auxquelles prennent part environ 20000 personnes. Pol.is ${ }^{41}$ leur permet d'exprimer leurs opinions et de réagir à celles des autres. La particularité de cet outil, dont le slogan est «Un meilleur discours public grâce à l'intelligence artificielle $\rightarrow$ des fonctionnaires mieux informés $\rightarrow$ de meilleures lois ", est qu'il n'y est pas possible de commenter les opinions des autres. Cela permet d'orienter la discussion vers la délibération et le consensus productif plutôt que vers la critique au vitriol et la division, qui caractérisent les débats sur certains réseaux sociaux ${ }^{42}$. Pour engager la discussion, en réponse à une opinion publiée, l'utilisateur de pol.is peut seulement indiquer son accord, désaccord ou passer son tour. Cela permet de grouper et de visualiser les opinions et de trouver une base commune de discussion afin de réduire les désaccords et atteindre un consensus sur une question précise. Dans le cadre de Talk to Taiwan, pol.is permet de définir les questions à poser à l'invité politique et de

[41] Colin Megill est à l'origine de la start-up de Seattle qui a créé pol.is. Il a publié dans Medium. com un article présentant le succès de l'utilisation de pol.is à Taïwan (Megill, 2016). Le fonctionnement de pol.is est assez proche de celui de Remesh.

[42] Il faut préciser ici qu'en résonance au «rough consensus » de Larry Wall, le consensus est défini de façon souple et finalement peu éloignée du « dissensus délibératif » où les interlocuteurs sont invités à préciser leurs arguments et entendre ceux des autres, sans avoir nécessairement à les partager. 
réagir à ses réponses de façon collective et délibérative. Avec vTaiwan, qui conduit désormais régulièrement au passage de lois à l'Assemblée nationale, pol.is a été mis en application dans la résolution remarquée de débats épineux concernant la régulation d'Uber (Tang, 2016b) et d'Airbnb.

La plateforme répond à sa promesse d'inclusivité. Elle s'adapte en effet à tous les formats et pourrait recevoir plusieurs millions de participants. De plus, étant donné qu'en moyenne dix fois plus de personnes votent que ne laissent de commentaires, elle élargit considérablement la consultation par rapport aux réseaux sociaux ne reposant que sur le commentaire des internautes les plus volubiles. La plateforme est ainsi devenue incontournable pour le gouvernement. En juillet 2016, le nouveau Premier ministre a déclaré à un conseil des ministres que «toutes les questions nationales importantes devraient passer par un processus de type vTaiwan ». Cette déclaration est particulièrement frappante car elle indique qu'un des obstacles majeurs à l'extension de la démocratie délibérative dans le monde, à savoir sa circonscription à des questions locales et restreintes, pourrait être bientôt levé. La limite principale de vTaiwan repose néanmoins sur les inégalités d'accès à Internet et de compréhension des outils numériques. vTaiwan est une plateforme avant tout consacrée aux questions liées aux nouvelles technologies, et toutes les personnes concernées par ces questions ne manquent pas de compétences à cet égard. Si son utilisation s'institutionnalise au-delà des seules questions numériques, le gouvernement taïwanais devra s'efforcer de réduire la fracture numérique et d'éduquer les générations les plus anciennes à ces nouveaux outils. De plus, puisque les participants aux délibérations ne sont pas tirés au sort, il est important d'étudier qui y participe, et si ce ne sont pas toujours les plus diplômés et les plus intéressés à la politique qui mobilisent les innovations numériques. Comme le souligne Yves Sintomer, « la délibération est un travail performatif qui crée un artefact, l'opinion du peuple telle qu'elle pourrait être si les moyens de délibérer correctement étaient réunis » (Sintomer, 2011), et c'est ce qui en fait un outil de décision microcosmique remarquable. L'édition 2008 de l'enquête sur le changement social à Taïwan menée par l'Academia Sinica depuis trente ans nous avait en effet appris que

«ce sont les individus qui parlent politique avec leurs amis en ligne et qui diversifient les médias d'interaction sociale en ligne qui ont le plus de probabilité de prendre directement contact avec les législateurs et les élus en ligne et d'exprimer leurs vues politiques dans des espaces publics en ligne tels que forums, blogs et sites d'information » (Hsieh, Li, 2016).

Cette enquête corrobore les études démontrant que ce sont les citoyens déjà les plus informés, éduqués, motivés et politisés qui bénéficient le plus des possibilités offertes par le Net (Delli Carpini, Keeter, 2002 ; Brabham, 2008). La fracture digitale et politique tend ainsi à se reproduire également dans la participation citoyenne en ligne, à Taïwan comme ailleurs. 


\section{Conclusion : la démocratie est-elle en train de se réinventer à Taïwan ? Avec quel impact transnational ?}

Dans la mesure où Internet et la programmation sont inséparables de l'histoire de la démocratie taïwanaise, l'activisme des programmeurs dans le cas taïwanais invite à une réflexion stimulante sur le renouveau de la démocratie par l'innovation, la mobilisation populaire, et surtout la mobilisation des jeunes générations. Audrey Tang est une programmeuse militante particulièrement efficace dans l'exploration de l'Internet et des outils numériques dans le but de s'armer de nouveaux outils pour mener de vieux combats, voire pour la réinvention de la démocratie. Si elle pourrait bien y contribuer, c'est parce qu'elle appartient en effet à une génération de citoyens militants dont la culture politique est ancrée dans celle du web et qui en a intégré les valeurs et les principes, et parce qu'elle incarne de façon inédite la réunion de trois champs (théorique, technologique et politique) et des compétences associées rarement réunies en une seule personne (synthèse des théories et pratiques délibératives; théorisation, conception, programmation de dispositifs originaux; communication et efficacité politiques, etc.). Avec ses partenaires de g0v, elle bénéficie d'un contexte favorable de réceptivité sociale et politique de leurs actions. Le mouvement des Tournesols a en effet considérablement aggravé la crise de confiance dans les institutions, fragilisé la légitimité de l'action gouvernementale et facilité l'arrivée au pouvoir de nouvelles figures politiques indépendantes et d'une présidente DDP, contraintes de répondre aux demandes de démocratisation du processus décisionnel à Taïwan en cooptant les hackers citoyens de g0v ayant converti la jeunesse taïwanaise à la délibération. Pour preuve, une plateforme de délibération en ligne influe désormais de façon régulière et institutionnalisée sur le processus législatif. Les institutions sont passées à l'open data, et Audrey Tang a été nommée ministre fin août 2016 et est entrée en fonction en octobre de la même année. Sous l'impulsion des jeunes générations de citoyens pour lesquelles démocratie et nouvelles technologies sont indissociables, la société taïwanaise est en passe d'évoluer vers une société de citoyens hackers, où les citoyens peuvent décider de l'ordre des priorités (agenda settingl et créer euxmêmes leurs outils de participation plutôt que de se les faire imposer.

Si les dirigeants de Taïwan ne se limitent pas à des effets d'annonce, la mise en application de cet empowerment suprême des citoyens taïwanais ne peut être complète sans la conversion massive des cadres et fonctionnaires aux divers échelons administratifs. Or, une vague massive de formation avait d'ores et déjà débuté pendant l'été $2016^{43}$. La communauté open source de Taïwan, qui se nourrit des actions d'autres communautés dans le monde qui partagent leurs lignes

[43] Trois membres de g0v (dont Audrey Tang), conseillers au Conseil pour le développement national pour la plateforme Join.gov.tw, ont en effet commencé à l'été 2016 à former 36 futurs formateurs, dans le cadre d'un partenariat avec le Centre de recherche académique de l'e-gouvernance taïwanaise. 
de code sur GitHub entre autres, apporte ainsi une contribution importante à la communauté mondiale, dont l'organisation du sommet international g0v de l'open source à Taipei en 2016 (Chen, 2016) témoigne. Elle transforme également la jeune démocratie taïwanaise, en quête de reconnaissance et qui se veut exemplaire face à la Chine, en Asie et dans le monde entier : si elle parvient réellement à se réinventer, l'impact pourrait en effet traverser les frontières lentre pays, mais également entre réel et virtuel). C'est bien l'ambition d'Audrey Tang, qui travaillait lors de notre dernière rencontre physique à la création d'un espace de délibération compatible avec l'usage des casques pour réalité virtuelle. En me faisant contempler dans le casque les constellations et en réponse à une question sur la transnationalité des hackers, elle expliquait :

«De l'espace, on voit des lumières, on voit les activités humaines, on voit la géographie, le climat, l'Arctique. On voit les aurores boréales, etc., mais on ne voit pas les pays, n'est-ce pas? Je pense que la mentalité du hacker est profondément marquée par cette vision topologique de la Terre comme des espaces connectés et non comme un lieu. $»^{44}$

\section{Bibliographie}

Ackerman B., Fishkin J., 2004, Deliberation day, New Haven, Yale University Press.

Albrecht S., 2006, « Whose voice is heard in online deliberation?: A study of participation and representation in political debates on the internet », Information, Communication \& Society, 9 (1), p. 66-82.

Badouard R., 2014, « La mise en technologie des projets politiques. Une approche “orientée-design" de la participation en ligne », Participations, 8, p. 31-54.

Badouard R., 2016, «\#LoiTravail : que peuvent les mobilisations en ligne?», The Conversation, 23 mars 2016, https://theconversation.com/loitravail-quepeuvent-les-mobilisations-en-ligne-56649 (accès le 21/01/2017).

Barlow J., 1996, «ADeclaration of the Independence of Cyberspace », 8 février 1996, https://www.eff.org/fr/cyberspace-independence laccès le 21/01/2017).

Barry L., 2016, «VTaiwan: public participation methods on the cyberpunk frontier of democracy », Civic Hall, 11 août 2016, http://civichall.org/civicist/vtaiwandemocracy-frontier/ laccès le 21/01/2017).

Blondiaux L., Fourniau J.-M., 2011, « Un bilan des recherches sur la participation du public en démocratie : beaucoup de bruit pour rien ? Participations, 1 , p. 8-35.

Bohman J., 1996, Public Deliberation: Pluralism, Complexity, and Democracy, Cambridge (MA), MIT Press.

[44] Entretien du 21 mars 2016. 
Brabham D., 2008, «Crowdsourcing as a model for problem solving: an introduction and cases $\gg$, Convergence, 14 (1), p. 75-90.

Cardon D., 2010, La démocratie Internet : promesses et limites, Paris, Seuil.

Cardon D., Fouetillou G., Lerondeau C., Prieur, C., 2011, « Esquisse de géographie de la blogosphère politique », in F. Greffet (dir.), Continuerlalutte.com. Les partis politiques sur le web, Paris, Presses de Sciences Po, p. 73-94.

Castells M., 2012, Networks of Outrage and Hope: Social Movements in the Internet Age, Cambridge (UK), Polity Press.

Chan M., 2016, «Teen pop star Chou Tzu-yu's apology for waving Taiwan flag swayed young voters for DPP », South China Morning Post, 17 janvier 2016, http://www.scmp.com/news/china/policies-politics/article/1902195/teenpop-star-chou-tzu-yus-apology-waving-taiwan-flag laccès le 21/01/2017).

Chen S., 1998, « State, Media and Democracy in Taïwan », Media Culture \& Society, 20 (1), p. 11-31.

Chen Y.-C., 2016, «Can Technology Improve Democracy? Answers from g0v Summit $2016 »$, Medium.com, 20 juillet 2016, https://medium.com/g0v-tw/ can-technology-improve-democracy-answers-from-g0v-summit-2016-db51a126e110\#. ipjzoqv1p (accès le 21/01/2017).

Chuang Y.-H., 2016, « Tournesols taïwanais, parapluies hongkongais. Occupy en Asie de l'Est », La Vie des idées, 22 septembre 2016, http://www.laviedesidees. $\mathrm{fr} /$ Tournesols-taiwanais-parapluies-hongkongais.html (accès le 21/01/2017).

Delli Carpini M., Keeter S., 2002, «The internet and an informed citizenry », in D. Anderson, M. Cornfield (dir.), The civic web, Lanham (MD), Rowman \& Littlefield.

Diamond L., Shin G.W., 2014, New challenges for maturing democracies, Korea and Taïwan, Stanford, Stanford University Press.

Ermonshina K., 2016, «Is there an App for everything? Potentials and limits of civic hacking », Observatorio, 11, p. 116-140.

Fishkin J., Luskin R., 1999, «Bringing Deliberation to the Demo Dialogue », in M. McCombs, A. Reynolds (dir.), The Poll with A Human Face National Issues Convention Experiment in Political Communication, Mahwat (NJ)/Londres, Lawrence Erlbaum Associates, p. 241-270.

Fishkin J., 2009, When the people speak, Oxford, Oxford University Press.

Flichy P., 2001, L'imaginaire d'Internet, Paris, La Découverte.

Flichy P., 2008a, «Internet, un outil de la démocratie ?», La Vie des idées, 14 janvier 2008, http://www.laviedesidees.fr/Internet-un-outil-de-la-democratie.html laccès le 21/01/2017).

Flichy P., 2008b, « Internet et le débat démocratique », Réseaux, 150, p. 159-185.

Fouetillou G., 2008, « Le web et le traité constitutionnel européen : écologie d'une localité thématique compétitive », Réseaux, 147, p. 159-185.

Frenkiel É., 2016, « Civic Hacking and our Political Future. An Interview with Audrey Tang », Books and Ideas, 25 février 2016, http://www.booksandideas. net/Civic-Hacking-and-our-Political-Future.html laccès le 21/01/2017). 
Gastil J., 1993, Democracy in Small Groups: Participation, Decision Making and Communication, Philadelphia, New Society Publishers.

Greffet F., Wojcik S., 2008, « Parler politique en ligne », Réseaux, 150, p. 19-50.

Gregg M., 2015, « Hack for good, speculative labour, app development and the burden of austerity », The Fibreculture Journal, 25, p. 183-201, http://twentyfive. fibreculturejournal.org/fcj-186-hack-for-good-speculative-labour-app-development-and-the-burden-of-austerity/ laccès le 21/01/2017).

Gutmann A., Thompson D., 2004, Why Deliberative Democracy?, Princeton, Princeton University Press.

Habermas J., 1998, Between facts and norms, Cambridge, MIT Press.

Hsieh Y., Li M.-H., 2016, « Online political participation, civic talk and media multiplexity: how Taïwanese citizens express political opinions on the Web», in W. Chen, The internet, social networks and civic engagement in civic societies, Londres/New York, Routledge, www.yulipatrickhsieh.org/wp-content/ uploads/2013/09/ICSText081213.pdf laccès le 21/01/2017).

Hsiao H., Wan P.-S., 2016, « 2014 Students Movements and Public Opinion: Taiwan and Hong Kong in Comparison », in H. Hsiao, S. Chiu, P.-S. Wan (dir.), Youth and Social Change in Taiwan and Hong Kong, Hong Kong, The Hong Kong Institute of Asia-Pacific Studies, The Chinese University of Hong Kong.

Huang T.-Y., Hsieh C.-A., 2013, « Practicing deliberative democracy in Taiwan: processes, impacts and challenges », Taïwan journal of democracy, 9 (2), p. 79-104.

Jensen L., 2003, «Public Spheres on the Internet: Anarchic or Governmentsponsored-a Comparison », Scandinavian Political Studies, 26 (4), p. 349-374.

Kittur A., Chi E. H., Suh B., 2008, « Crowdsourcing User Studies with Mechanical Turk », in Proceedings of the SIGCHI conference on human factors in computing systems, Florence (Italie), 5-10 avril 2008, p. 453-456.

Lee Y., 2014, «Korea's economic transformation since 1997 » in L. Diamond, G.W. Shin, New challenges for maturing democracies, Korea and Taïwan, Stanford, Stanford University Press.

Lev-On A., Manin B., 2006, « Internet : la main invisible de la délibération », Esprit, mai 2006, http://www.esprit.presse.fr/article/lev-on-azi-et-manin-bernard/ internet-la-main-invisible-de-la-deliberation-13254 laccès le 21/01/2017).

Lewanski R., 2013, «Institutionalizing deliberative democracy: the Tuscany “laboratory" », Journal of Public Deliberation, 9 (1), http://www.publicdeliberation.net/cgi/viewcontent.cgi?article=1185\&context=jpd (accès le 21/01/2017).

Lin I.F., 2014, « How technology and citizen media shaped Taiwan's Sunflower Movement », Global Voices, 20 avril 2014, https://globalvoices.org/2014/04/20/ how-technology-and-citizen-media-shaped-taiwans-sunflower-movement/ laccès le 21/01/2017).

Megill C., 2016, « Pol.is in Taiwan », Medium.com, 25 mai 2016, https://blog.pol.is/ pol-is-in-taïwan-da7570d372b5\#.9csjl6hku laccès le 21/01/2017).

Meyer M., Ermoshina K., 2013, « Bricolage as collaborative exploration: transforming matter, citizens and politics », i3 Interdisciplinary Innovation Conference, Paris, Mines ParisTech, 2 décembre 2013. 
Monnoyer-Smith L., 2006, «Être créatif sous la contrainte : Une analyse des formes nouvelles de la délibération publique. Le cas DUCSAI », Politix, 3 (75), p. 75-101.

Morozov E., 2009, « Foreign policy: brave new world of slacktivism », NPR, 19 mai 2009, www.npr.org/templates/story/story.php?storyld=104302141 laccès le 21/01/2017).

Pai C.-F., 2010, « Deliberative democracy via cyberspace: a study of online political forums QिTaiwan », thèse de doctorat, Cardiff University.

Pariser E., 2011, The Filter bubble: What the Internet is hiding from you, New York, Penguin.

Rheingold H., 1993, The virtual community: homesteading on the electronic frontier, Reading (MA), Addison Wesley.

Richard C., 2015, «Audrey Tang, programmeuse géniale, hacke la politique à Taïwan », Rue89, 28 septembre 2015, http://rue89.nouvelobs.com/2016/08/25/ audrey-tang-programmeuse-geniale-hacke-politique-a-taïwan-261381 laccès le 21/01/2017).

Rosanvallon P., 2006, La Contre-démocratie, Paris, Seuil.

Rothwell S., Carter S., Elshenawy A., Braga D., 2016, « Job Complexity and User Attention in Crowdsourcing Microtasks », Crowdsourcing Breakthroughs for Language Technology Applications, AAAI Technical Report WS-15-24, http://www.aaai.org/ocs/index.php/HCOMP/HCOMP15/paper/viewFile/11735/12334 (accès le 21/01/2017).

Shirky C., 2012, « How the internet will lone dayl transform government », TED Global, juin 2012, https://www.ted.com/talks/clay_shirky_how_the_internet_ will_one_day_transform_government?language=en (accès le 21/01/2017).

Sintomer S., 2011, Petite histoire de l'expérimentation démocratique, Paris, La Découverte.

Sunstein C., 2001, Republic.com, Princeton, Princeton University Press.

Sunstein C., 2007, Republic.com 2.0., Princeton, Princeton University Press.

Tang A., 2016a, «Challenges for civic hackers in 2016 », Medium.com, 3 janvier 2016, https://medium.com/daudrey.tang/challenges-for-taiwan-scivic-hackers-in-2016-385af61d6e79\#.Isnnjac04 (accès le 21/01/2017).

Tang A., 2016b, « Uber responds to vTaiwan's coherent blended volition », pol.is blog, 23 mai 2016, https://blog.pol.is/uber-responds-to-vtaïwans-coherentblended-volition-3e9b75102b9b\#. ubbzpd6es laccès le 21/01/2017).

Tang A., 2016c, « Resolving singularities », Medium.com, 25 mai 2016, https://medium.com/Qaudrey.tang/resolving-singularities-bb247e2af10a\#.1kkas4735 laccès le 21/01/2017).

Tang A., 2016d, « Une expérience pionnière de démocratie numérique à Taïwan », Le Monde, 25 mai 2016, http://www.lemonde.fr/idees/article/2016/05/25/uneexperience-pionniere-de-democratie-numerique-a-taiwan_4926104_3232. html\#0xxKqXhL5rkzRvm0.99 (accès le 21/01/2017). 
Tsai I.-W., 2016, Full text of President Tsai's inaugural address, 20 mai 2016, http:// focustaiwan.tw/news/aipl/201605200008.aspx (accès le 21/01/2017).

Tso C.-D., «Digital media and the transformation of politics in Taiwan », in L. Diamond, G.W. Shin, 2014, New challenges for maturing democracies, Korea and Taiwan, Stanford, Stanford University Press.

Wall L., Christiansen T., Schwartz R., 1996, Programming Perl, $2^{\mathrm{e}}$ édition, Sebastopol (CA), 0'Reilly \& Associates.

Wang C, Shih H.-C., 2012, «Cabinet ad pulled from Youtube», Taipei Times, 18 octobre 2012, http://www.taipeitimes.com/News/front/archives/2012/10/18/2003545458 (accès le 21/01/2017).

Warren M., 1996a, «Deliberative democracy and authority », American political science review, 86, p. 8-23.

Warren M., 1996b, « What we should expect from more democracy. Radical democratic responses to politics », Political Theory, 24, p. 241-270.

Wright S., Street J., 2017, «Democracy, deliberation and design: the case of online discussion forums », New Media \& Society, 9 (5), p. 849-969.

\section{Abstract - Hacking Democracy: Audrey Tang and the Reinvention of Taiwanese Politics}

This article is devoted to Audrey Tang, who belongs to the young civic hacker group g0v and who became the minister in charge of digital affairs in Taiwan in October 2016. It investigates how digital participatory devices are designed in the Taiwanese context, which is at a crossroads of Western and Chinese influences and has been involved in a process of thorough political renewal since the student occupation of Parliament during the 2014 Sunflower Movement. The article examines the causes for this uncommon encounter between technical know-how, theoretical reflection, and political will.

Keywords Taiwanese Democracy, Civic Tech, Online Deliberation, Youth Participation, Hacker 
Emilie Frenkiel est maître de conférences à l'UPEC et chercheuse au LIPHA. Elle est docteur en étude politique de l'EHESS. Ses recherches portent sur la représentation et la participation politiques en Chine, et tout particulièrement sur les innovations participatives mises en place par les autorités locales en Chine. Elle a notamment publié Parler politique en Chine, Presses universitaires de France, 2014 et Conditional democracy, The contemporary debate on political reform in Chinese universities, ECPR Press, 2015. Elle est co-rédactrice en chef de La Vie des Idées et Books\&ldeas.

\section{Mots clés}

Démocratie taïwanaise, civic tech, délibération en ligne, participation politique des jeunes, Hacker 